\title{
Decompression Process of Glycerol Shock Treatment Can Overcome Endo-Lysosomal Barriers for Intracellular Delivery
}

\author{
Shupeng Wang, Shaohua Jin, Guangzhi Li, Rui Sun, Qinghai Shu,* and Song Wu* \\ Cite This: ACS Omega 2020, 5, 33133-33139 \\ Read Online
}

ABSTRACT: The glycerol shock treatment has been used to improve the calcium phosphate transfection efficacy for several decades because of its high effectiveness and low toxicity. However, the mechanism of glycerol shock treatment is still obscure. In this study, the endo-lysosomal leakage assay demonstrated that the decompression process of glycerol shock treatment could enhance endo-lysosomal membrane permeabilization, which resulted in facilitating endo-lysosomal escape for effective intracellular delivery. The enhanced decompression treatment derived from glycerol shock treatment could increase the change of osmotic pressure further, which showed higher efficacy for intracellular delivery. Herein, we speculated that the endo-lysosomal swelling originated from the decompression process of glycerol shock treatment could cause endolysosomal damage.

A $15 \%$ glycerol solution

Culture medium

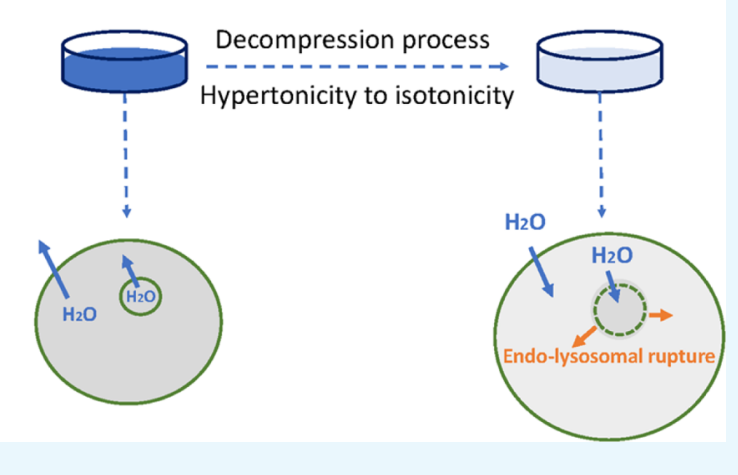

\section{INTRODUCTION}

In 1973, Graham and van der Eb found that cells could be transfected with herpes simplex virus DNA by the calcium phosphate $(\mathrm{CaP})$ precipitate. ${ }^{1}$ This research initiates the widespread application of $\mathrm{CaP}$ transfection for introducing foreign DNA into cultured cells. Jordan et al. subsequently have optimized a CaP transfection method, including glycerol shock treatment, which refers to the operation where cells are transfected with the CaP/DNA precipitate for several hours first, following the removal of the precipitate, and then the cells are exposed to about $15 \%(\mathrm{v} / \mathrm{v})$ glycerol solution for several minutes. $^{2-4}$ This method is modified by widely used Cold Spring Harbor protocols, which state that the uptake of DNA could be increased after glycerol shock treatment. ${ }^{5}$ However, the mechanism of how glycerol shock treatment could increase the uptake of DNA after removal of the CaP/DNA precipitate is still not clear. In this study, we attempted to explore the underlying mechanism of glycerol shock treatment.

Glycerol has been widely used in the biomedical field, for instance, as an osmotic agent to treat cerebral edema or a cryoprotective agent. ${ }^{6,7}$ The history of glycerol shock treatment has been shown in Figure S1. As previously reported, Jordan confirmed a glycerol shock did not affect the uptake of DNA into the cells in $\mathrm{CaP}$ transfection by labeled DNA.,4 They speculated that the shock treatment appeared to act on cell-endocytosed DNA, which might favor the conjecture that DNA might escape from endo-lysosomes, but no direct evidence was provided. Until now, glycerol shock treatment has been used in $\mathrm{CaP}$ transfection for about 4 decades; could more direct evidence explain the mechanism?
In the present study, we have demonstrated that the decompression process of glycerol shock treatment can enhance endo-lysosomal membrane permeabilization to promote endolysosomal escape. As shown in Scheme 1, when cells are exposed to a $15 \%$ glycerol solution for several minutes, endo-lysosomal membrane permeabilization does not change. Then, cells return to an isotonic solution, the osmotic pressure is from hypertonicity to isotonicity, and the endo-lysosomal membrane permeabilization can be enhanced significantly. Therefore, not the hypertonic glycerol solution but the decompression process from hypertonicity to isotonicity mainly contributed to enhancing endo-lysosomal membrane permeabilization. Glycerol shock treatment also might be applied to other areas related to endo-lysosomal membrane permeabilization, such as autophagy and cancer therapy. ${ }^{8-10}$

\section{RESULTS AND DISCUSSION}

Effect of Glycerol or $\mathrm{NaCl}$ Shock Treatment on CaP Transfection. The mechanism of glycerol shock treatment in $\mathrm{CaP}$ transfection has long been a mystery. Jordan et al. demonstrated that glycerol shock treatment could not enhance the uptake of $\mathrm{CaP} / \mathrm{DNA},{ }^{3,4}$ we also verified that the shock treatment did not improve cellular uptake efficacy of YOYO-1-

Received: September 29, 2020

Accepted: October 28, 2020

Published: December 16, 2020 
Scheme 1. Schematic Illustration Showing the Glycerol Shock Treatment for Intracellular Delivery

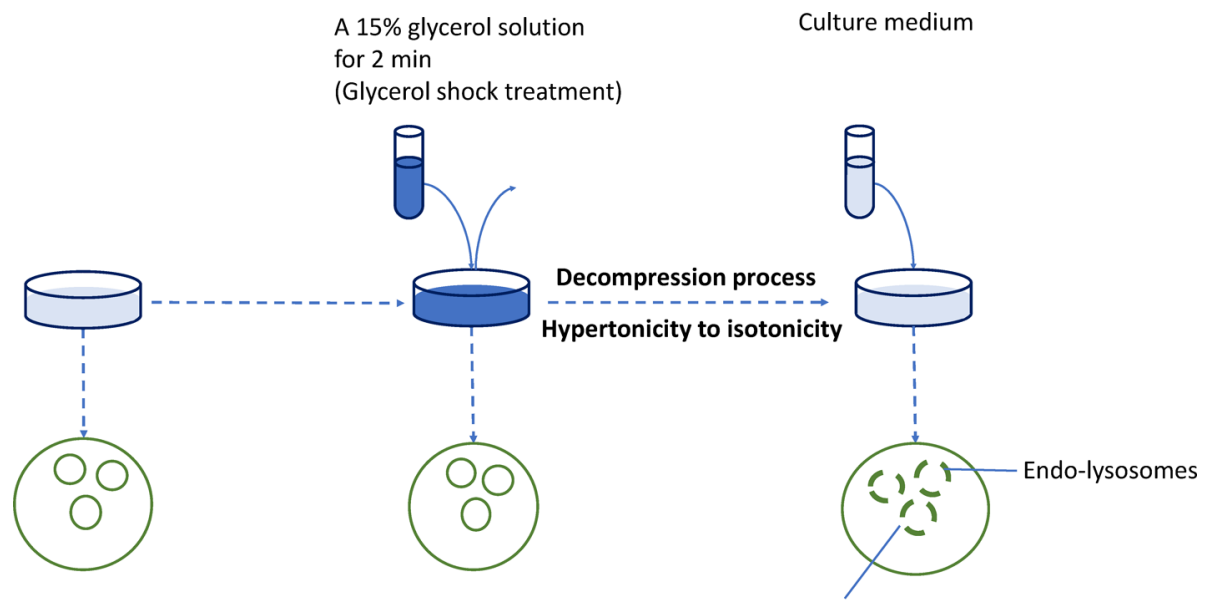

Overcoming endo-lysosomal barriers

A
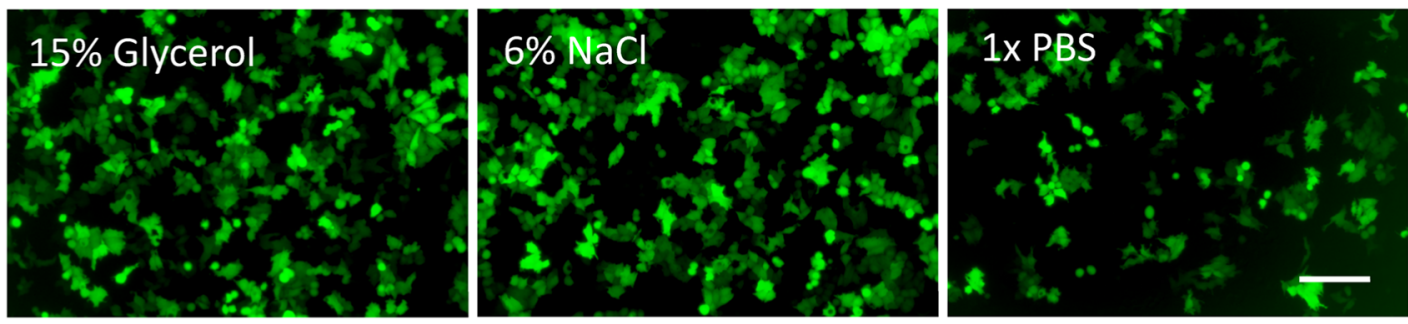

B

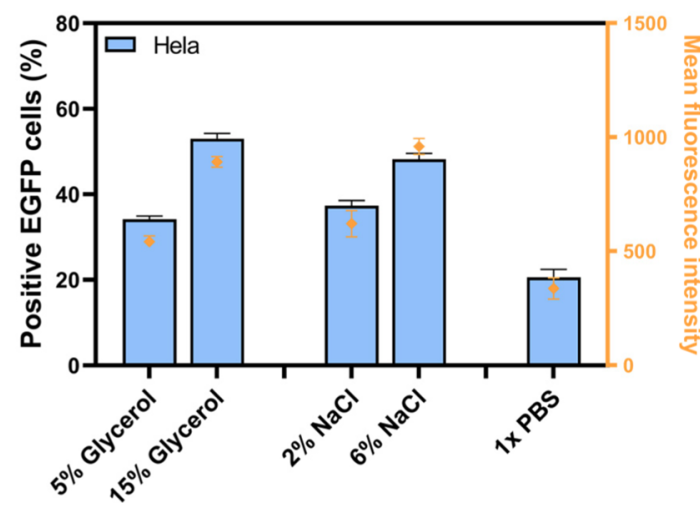

C

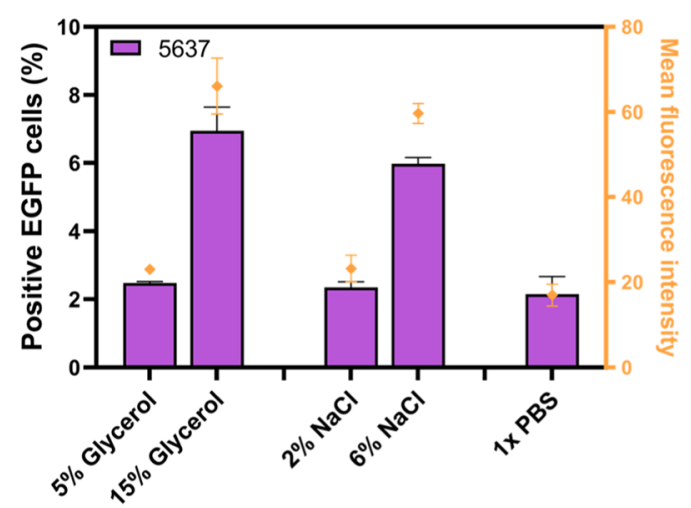

Figure 1. Transfection efficacy of $\mathrm{CaP}$ transfection in Hela and 5637 cells. Comparison of glycerol shock and $\mathrm{NaCl}$ shock treatment on transfection efficacy in (A,B) Hela cells and (C) 5637 cells after 48 h, respectively. Control is the group treated with $1 \times$ PBS. Scale bar, $200 \mu \mathrm{m}$.

labeled DNA in CaP transfection (Figure S2), so the shock treatment might influence other processes of transfection. As previously reported, either glycerol or dimethyl sulfoxide shock treatment could enhance CaP transfection effectively. ${ }^{11,12}$ Moreover, it was reported that polyethylene glycol shock also could improve transfection efficiency. ${ }^{13}$

We speculated that glycerol shock treatment was related to hypertonicity; to test our hypothesis, we performed $\mathrm{NaCl}$ shock treatment. We observed that either glycerol or $\mathrm{NaCl}$ shock treatment improved the transfection efficacy of $\mathrm{CaP}$ transfection, which was verified in two different cell lines, Hela and 5637 cells (Figures 1 and S3). For Hela cells, the percentage of positive enhanced green fluorescent protein (EGFP) cells increased from 20.7 to $53.1 \%$ after a $15 \%$ glycerol shock and from 20.7 to $48.2 \%$ after a $6 \% \mathrm{NaCl}$ shock. For 5637 cells, the percentage of positive EGFP cells increased from 2.15 to $6.97 \%$ after a $15 \%$ glycerol shock and from 2.15 to $5.98 \%$ after a $6 \%$ $\mathrm{NaCl}$ shock. The findings suggested that improved transfection efficacy might be highly related to hypertonicity. Next, we investigated the role of hypertonic shock treatment in transfection.

Effect of Hypertonic Glycerol Shock Treatment on Endo-Lysosomes. Generally, endo-lysosomal entrapment is a significant barrier to effective intracellular DNA delivery, ${ }^{14-16}$ which can be observed by the intracellular distribution of labeled DNA (a green fluorescent dye, YOYO-1) and LysoTracker Redstained endo-lysosomes. Here, we investigated the effect of glycerol shock treatment on the intracellular DNA and endo- 
A

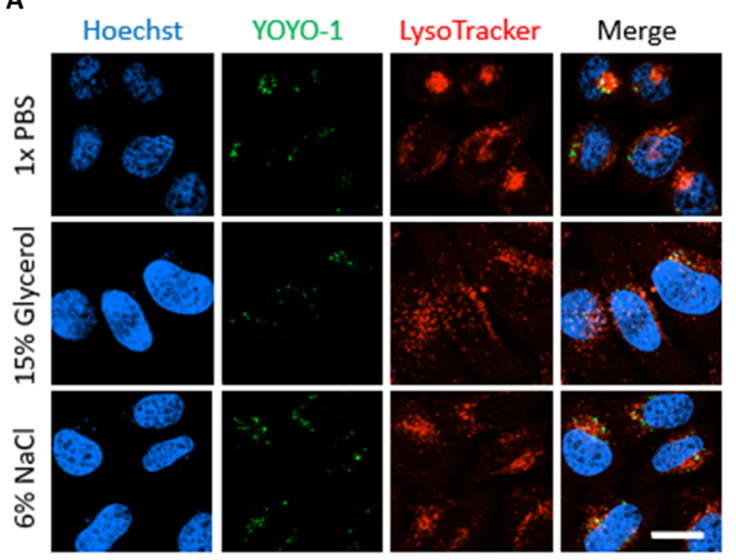

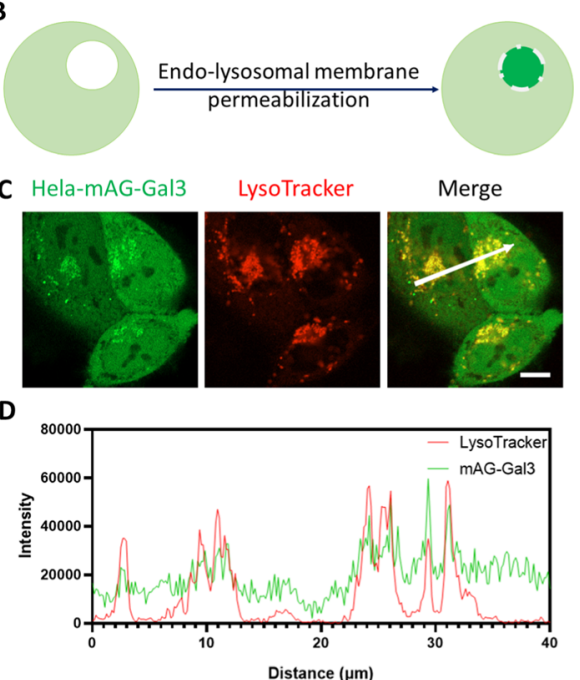

Figure 2. Effect on hypertonic shock treatment on endo-lysosomes. (A) CLSM images of Hela cells treated with CaP/DNA for $4 \mathrm{~h}$ before a $15 \%$ glycerol or $6 \% \mathrm{NaCl}$ shock, respectively. Control is the group treated with $1 \times \mathrm{PBS}$. Scale bar: $20 \mu \mathrm{m}$. (B) Schematic representation of the endolysosomal leakage assay. The permeabilization of endo-lysosomes allows the entry of cytosolic mAG-Gal3 proteins, leading to bright fluorescent spots. (C) Hela-mAG-Gal3 cells treated with a 15\% glycerol shock. Glycerol shock treatment made mAG-Gal3 accumulate in ruptured endo-lysosomes, and bright fluorescent spots showed high colocalization with endo-lysosomes. Scale bar: $10 \mu \mathrm{m}$. (D) Intensity of red and green fluorescence on the arrow line in (C), mAG-Gal3: green, LysoTracker: red. Scale bar: $20 \mu \mathrm{m}$.

lysosomes. As shown in Figure 2A, LysoTracker Red-stained endo-lysosomes were more disperse after the shock treatment, which indicated that hypertonic shock treatment (a 15\% glycerol shock or a $6 \% \mathrm{NaCl}$ shock) appeared to have more impact on endo-lysosomes than YOYO-1-labeled DNA. These results suggested that glycerol shock treatment might destabilize and destroy endo-lysosomes.

Endo-Lysosomal Leakage Assay. To further confirm glycerol shock treatment has an impact on endo-lysosomes, we applied Gal-3 reporter cells to perform the endo-lysosomal leakage assay for more clear evidence. The Gal-3 reporter system has been used to visualize macropinosome leakage ${ }^{17}$ and lysosomal membrane permeabilization. ${ }^{18}$ In this system, Gal-3 is a soluble protein that generally existed in the cytosol, which can bind $\beta$-galactoside sugar-containing carbohydrates. ${ }^{19}$ These carbohydrates are usually present on the interior of endocytic vesicles. The permeabilization of vesicles results in cytosolic mAG-Gal-3 being accessible to carbohydrates and accumulating at ruptured vesicles, and fluorescent spots can be observed (Figure 2B). ${ }^{17}$

As shown in Figure 2C,D, glycerol shock treatment made Hela-mAG-Gal3 cells produce bright fluorescent spots, which showed high colocalization with endo-lysosomes. In addition, we performed siramesine (a lysosomal membrane permeabilization inducer ${ }^{18}$ ) treatment, and either effect of glycerol shock or siramesine treatment was distinct (Figure S4). The result indicated that the endo-lysosomal leakage assay was an excellent tool to visualize endo-lysosomal membrane permeabilization. ${ }^{18,19}$ This result also suggested that glycerol shock treatment could overcome endo-lysosomal barriers by enhancing endolysosomal membrane permeabilization.

Decompression Process in Glycerol Shock Treatment. Inspired by the results above, we noticed that a glycerol shock could be divided into two procedures. The first procedure is to expose cells to a hypertonic glycerol solution. The second procedure is to recover isotonicity (as shown in Figure 3A). To determine each procedure's role in a hypertonic glycerol shock, we used Hela-mAG-Gal3 and 5637-mAG-Gal3 cells to perform the endo-lysosomal leakage assay. Contrary to our expectations, endo-lysosomal membrane permeabilization usually occurred in the second procedure, not the first procedure. As shown in Figure 3B,C, when Hela cells were exposed to a $15 \%$ glycerol or $6 \% \mathrm{NaCl}$ solution, the endo-lysosomal membrane permeabilization did not change; few fluorescent spots were observed. However, when cells were exposed to an isotonic solution again, many fluorescent spots appeared immediately. The phenomenon was also observed in 5637 cells (Figure S5).

The endo-lysosomal change caused by the decompression process may be similar to the post-hypertonic injury of human spermatozoa. ${ }^{20}$ The hypertonic solution makes sperm severely shrink, but the sperm appears to maintain membrane integrity in the hypertonic solution. Severe membrane damage only be detected after the sperm returns to isotonic conditions. ${ }^{20}$ The hypertonic glycerol or $\mathrm{NaCl}$ solution can cause cells to lose water to shrink..$^{20,21}$ Because of intrinsic membrane permeability, endo-lysosomes are affected by the ambient hyperosmotic environment, causing dehydration and shrinking. ${ }^{22}$ When cells are exposed to an isotonic solution again, endolysosomes will swell theoretically. In this study, we found that the endo-lysosome appeared to maintain membrane integrity in the shrunken state. The permeability of the endo-lysosomal membrane was enhanced after the cell was in isotonic conditions again.

Maintaining membrane integrity in the shrunken state may be the character of a biological membrane responding to the pressure change. However, irreversible changes may happen on the membrane during the dehydration process. The membrane could be damaged in the reswell process. We speculated that the endo-lysosomal swelling originated from the decompression process of glycerol shock treatment could cause endo-lysosomal damage and rupture (Figure 3D).

Enhanced Decompression Treatment in CaP Transfection. Furthermore, how to improve endo-lysosomal membrane permeabilization for higher transfection? Only 


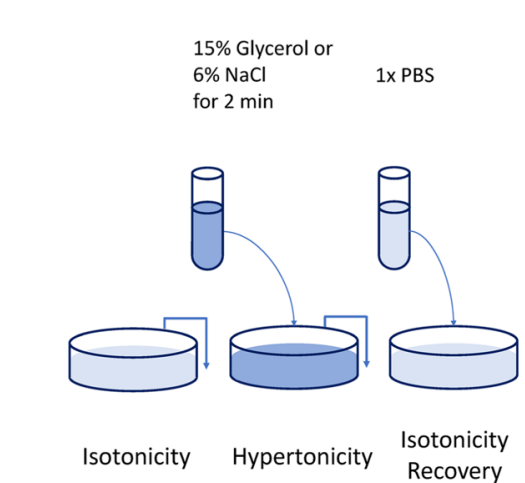

D
B

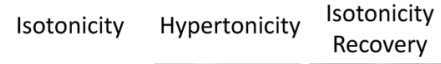

C

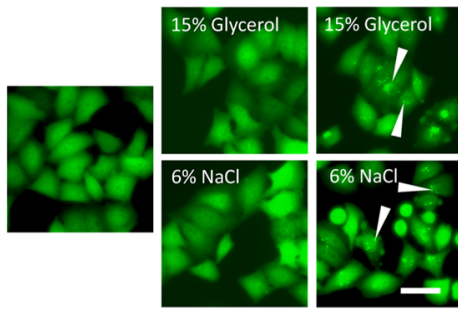

Isotonicity Hypertonicity Isotonicity
Recovery
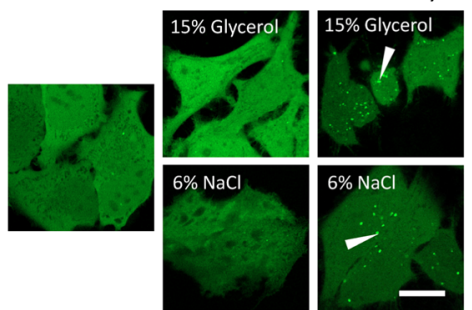

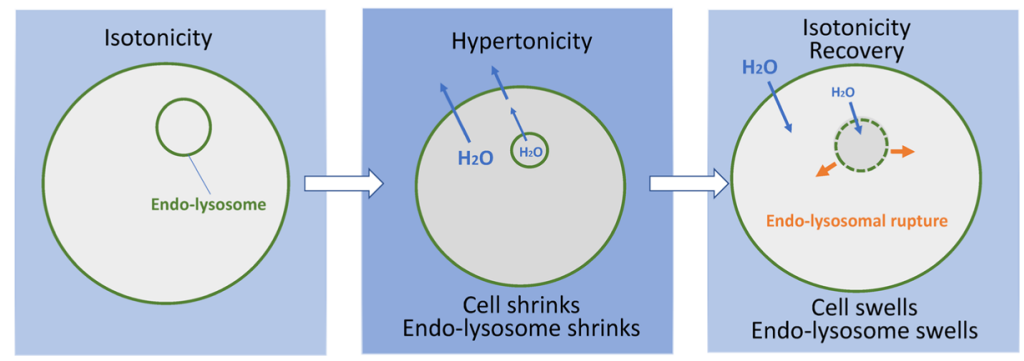

Figure 3. Endo-lysosomal leakage assay. (A) Schematic representation of a hypertonic shock. A hypertonic shock includes two steps. The first step is from isotonicity to hypertonicity. The second step is to return to isotonicity. (B) Fluorescence images of Hela-mAG-Gal3 cells treated with a 15\% glycerol shock or a $6 \% \mathrm{NaCl}$ shock. The decompression process enhanced endo-lysosomal membrane permeabilization. The arrows indicate ruptured endo-lysosomes. Scale bar: $50 \mu \mathrm{m}$. (C) CLSM images of Hela-mAG-Gal3 cells treated with hypertonic shock treatment. Scale bar: $20 \mu \mathrm{m}$. (D) Proposed mechanisms of decompression treatment. The dark background represents the hypertonic state; the light background represents the hypotonic state. The hypertonic solution can cause cells to lose water to shrink. Endo-lysosomes are also affected by the cell's hyperosmotic environment, causing dehydration and shrinking. However, endo-lysosome still appeared to maintain membrane integrity. Once cells return to an isotonic solution, endo-lysosomes will swell because of the influx of water; eventually, endo-lysosomes rupture.

increasing the concentration of a glycerol solution is not a good idea, which may kill cells because of severe dehydration. As shown in Figure 4A, cells and endo-lysosomes lose water to shrink in a hypertonic environment. The exchange from a hypertonic solution to an isotonic solution leads to an increase in the influx of water to maintain osmolarity. The influx of water could make the endo-lysosome swell and eventually cause the endo-lysosomal rupture. The exchange from a hypertonic solution to a hypotonic solution makes more water rush into endo-lysosomes, which could be damaged by more intense swelling. Herein, we proposed that the enhanced decompression treatment, a hypertonic glycerol treatment combined with a hypotonic treatment, might improve endo-lysosomal membrane permeabilization for higher transfection efficacy.

To test our hypothesis that the enhanced decompression treatment could achieve higher transfection efficacy than conventional glycerol shock treatment, we investigated the effects of enhanced decompression treatment in CaP transfection. Here, hypertonic glycerol shock treatment included a 15 or $5 \%$ glycerol shock. Hypotonic shock treatment included a series of dilute Dulbecco's modified Eagle medium (DMEM) medium (0.6× DMEM refereed to six parts DMEM to four parts water). As shown in Figure $4 \mathrm{~B}$, the combined treatment of a hypertonic glycerol shock and a hypotonic shock was more effective than only hypertonic glycerol shock treatment. The treatment of a $15 \%$ glycerol shock treatment combined with a 0.6× DMEM hypotonic shock treatment achieved the highest transfection efficacy. Cells with reduced viability may gain a relatively low level of transfection efficiency. The lower percentage of EGFP positive cells of group $0.4 \times$ DMEM than the group $0.6 \times$ DMEM may be attributed to the decreased cell viability of group $0.4 \times$ DMEM (Figure $4 \mathrm{C}$ ). These results suggested that enhanced decompression treatment is an efficient and biocompatible method to enhance transfection in $\mathrm{CaP}$ transfection.

Decompression Treatment in Chitosan/DNA Delivery. Inspired by the results above, decompression treatment was applied to improve chitosan/DNA transfection efficiency. The group of a $15 \%$ glycerol shock combined with a $0.6 \times$ DMEM hypotonic shock also achieved excellent gene transfection efficacy. The percentage of EGFP-positive cells of the group above was 8.6-fold higher than the group without shock treatment (Figure 5). The results suggested that endo-lysosomal escape was a limited step in chitosan/DNA delivery, which also could be promoted by glycerol shock treatment. 
A

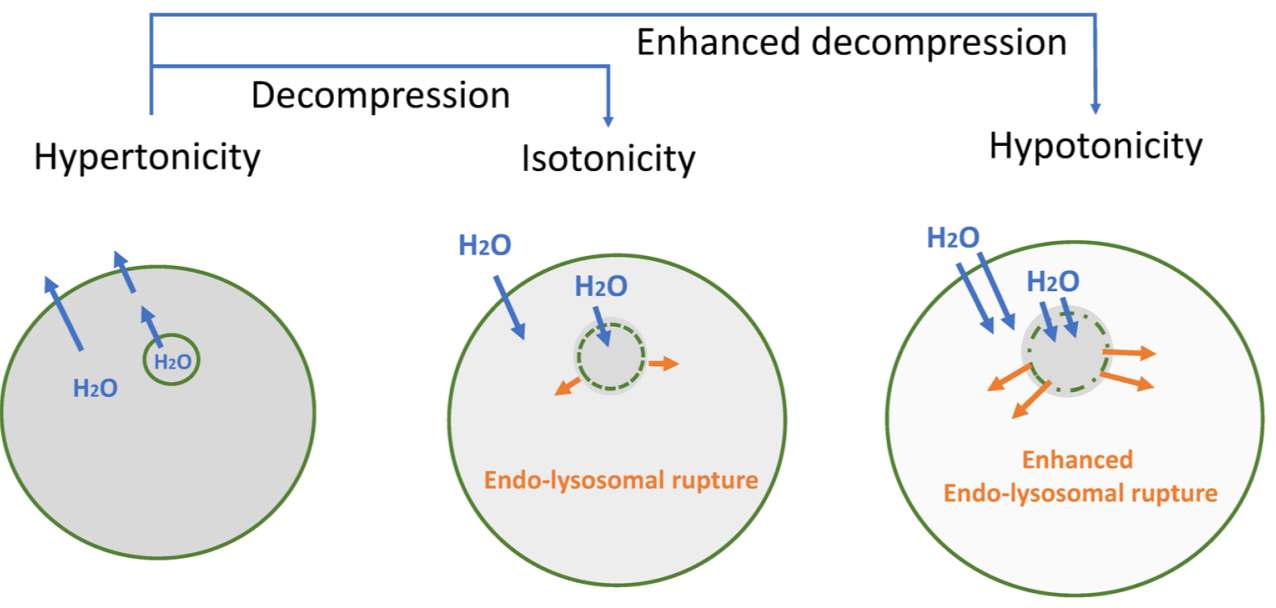

B

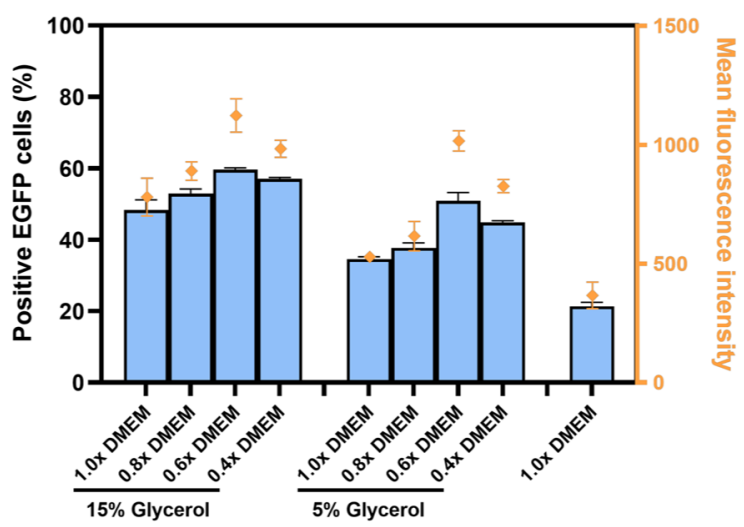

C

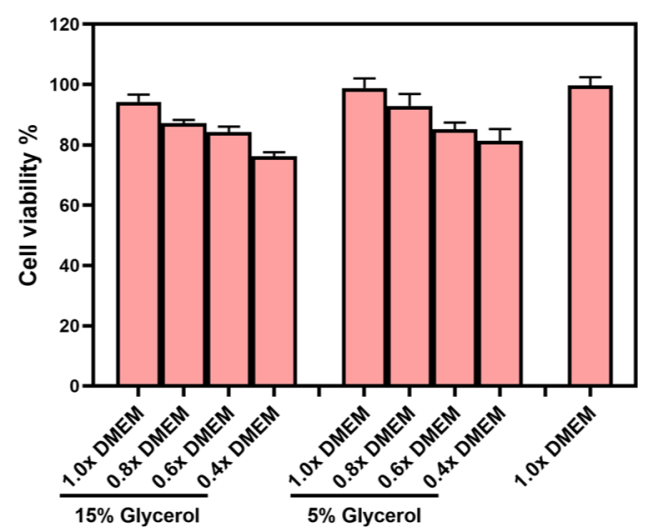

Figure 4. Enhanced decompression treatment. (A) Schematic representation of enhanced decompression treatment. Cells and endo-lysosomes will lose water to shrink in a hypertonic environment. The exchange from a hypertonic solution to an isotonic solution will lead to an increase in the influx of water. The influx of water makes the endo-lysosome swell to rupture. The exchange from a hypertonic solution to a hypotonic solution makes more water rush into endo-lysosomes, which could be damaged by more intense swelling. (B) Transfection efficacy of CaP transfection with enhanced decompression treatment in Hela cells. (C) Effects of enhanced decompression treatment on cell viability.

\section{A}

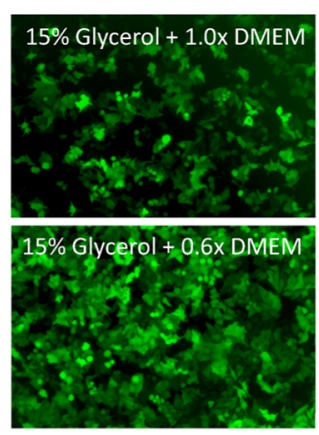

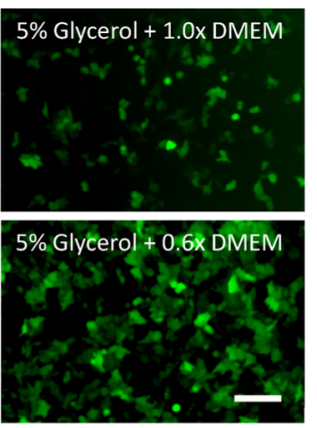

\section{B}

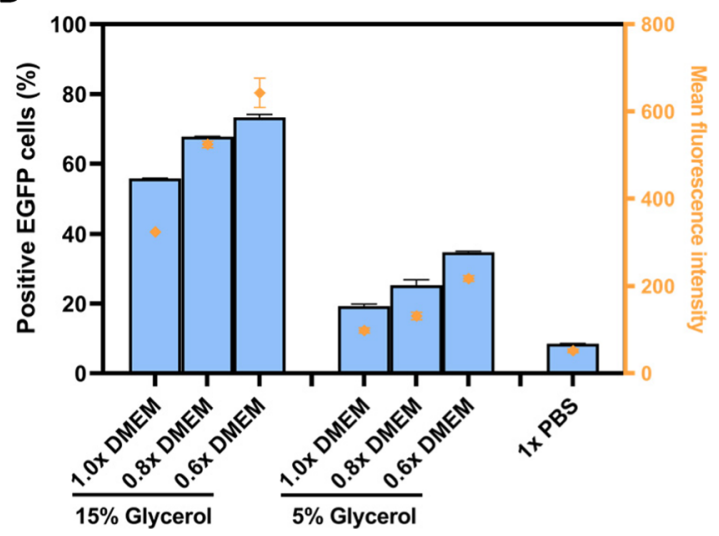

Figure 5. Decompression treatment in chitosan/DNA delivery. (A) Fluorescence images of chitosan/DNA delivery combined with decompression treatment in Hela cells. Scale bar: $200 \mu \mathrm{m}$. (B) Transfection efficacy of chitosan/DNA delivery combined with decompression treatment in Hela cells.

\section{CONCLUSIONS}

In this work, we showed the newly observed mechanism of glycerol shock treatment for intracellular delivery. The endolysosomal leakage assay provided essential proofs, which demonstrated that the decompression process of glycerol shock treatment could enhance endo-lysosomal membrane permeabilization to promote endo-lysosomal escape for effective delivery. Moreover, we found that glycerol shock treatment, combined with a hypotonic shock, might further overcome endo-lysosomal barriers by more intense swelling. Gene transfection is a complicated biological process, and the efficiency of gene transfection may be affected by many factors. 
Future works will be focused on investigating other mechanisms of glycerol shock treatment on gene transfection. In a word, glycerol shock treatment is an efficient and easy-to-operate method to facilitate endo-lysosomal escape quickly, and this method may have applications in many areas related to endolysosomal membrane permeabilization.

\section{EXPERIMENTAL SECTION}

Materials. $\mathrm{CaCl}_{2}$, glycerol, HEPES, and $\mathrm{Na}_{2} \mathrm{HPO}_{4} \cdot 12 \mathrm{H}_{2} \mathrm{O}$ were purchased from Sigma (USA). YOYO-1, LysoTracker Red, and Hoechst 33342 were purchased from Invitrogen (USA). Chitosan ( $80 \%$ deacetylated, low-molecular weight) was purchased from TCI (Japan). $\mathrm{NaCl}$, cell counting kit-8 (CCK8 ) was obtained from Sangon Biotech (China). The chemicals were used as received without further purification.

Plasmid Amplification and Purification. Plasmid EGFP (5010 bp) was purchased from Beyotime Biotechnology (China). Plasmid mAG-Gal3 (9610 bp) was a gift from Niels Geijsen (Addgene plasmid \# 62734). ${ }^{17}$ Plasmid EGFP was amplified by Escherichia coli DH5 $\alpha$ (Sangon, China), and plasmid mAG-Gal3 was amplified by E. coli Stbl3 (Sangon, China) according to the manufacturer's protocols. The amplified plasmids were purified by using the HiPure Plasmid Maxi Kit (Magen, China) according to the product's protocol. The concentration and integrity of purified plasmids were confirmed by spectrophotometry (NanoDrop, USA) and DNA electrophoresis, respectively.

Cell Culture. Hela cells (a human cervical cancer cell line, ATCC) were cultured in DMEM (Gibco, USA) supplemented with $10 \%$ fetal bovine serum (FBS, Hyclone), $100 \mu \mathrm{g} / \mathrm{mL}$ streptomycin, and $100 \mathrm{U} / \mathrm{mL}$ penicillin at $37^{\circ} \mathrm{C}$ with $5 \% \mathrm{CO}_{2}$, and 5637 cells (a bladder cancer cell line, ATCC) were cultured in RPMI-1640 medium (Gibco, USA) supplemented with $10 \%$ FBS, $100 \mu \mathrm{g} / \mathrm{mL}$ streptomycin, and $100 \mathrm{U} / \mathrm{mL}$ penicillin at 37 ${ }^{\circ} \mathrm{C}$ with $5 \% \mathrm{CO}_{2}$.

Generation of Gal3 Reporter Cell Lines. The lentiviral particles were produced by a lentiviral transduction system containing the plasmid mAG-Gal3. Hela-mAG-Gal3 and 5637$\mathrm{mAG}-\mathrm{Gal} 3$ reporter cell lines were made by infecting cells with the lentiviral particles. After 2 days of lentiviral infection, the cells were cultured in complete medium supplemented with 1 $\mu \mathrm{g} / \mathrm{mL}$ of puromycin at $37{ }^{\circ} \mathrm{C}$ with $5 \% \mathrm{CO}_{2}$. The addition of puromycin was used to select the cells that are stably expressing mAG-Gal3 proteins. The effect of lentiviral infection could be observed by fluorescent microscopy (Zeiss, Germany).

Calcium Phosphate Transfection. The cells were inoculated at $5 \times 10^{4}$ cells/well in 24-well plates for $24 \mathrm{~h}$ before transfection. $100 \mu \mathrm{L}$ of $2.5 \mathrm{M} \mathrm{CaCl}_{2}$ and $25 \mu \mathrm{g}$ of plasmid EGFP were adjusted to $1 \mathrm{~mL}$ with water; this $2 \times \mathrm{CaCl}_{2} / \mathrm{DNA}$ mix was added into an equal volume of $2 \times$ HEPES-buffered saline, as previously described. ${ }^{5}$ The CaP/DNA solution was incubated for $2 \mathrm{~min}$. The solution was immediately added to the medium. A $50 \mu \mathrm{L}$ of the solution was used for each $500 \mu \mathrm{L}$ of the medium in a well. After incubation for $4 \mathrm{~h}$ at $37^{\circ} \mathrm{C}$ and $5 \% \mathrm{CO}_{2}$, each well was washed with $1 \times$ PBS and supplemented with $500 \mu \mathrm{L}$ complete medium for another $42 \mathrm{~h}$ before transfection efficacy analysis.

Shock Treatment. After the $\mathrm{CaP} / \mathrm{DNA}$ precipitate was incubated with the cells for $4 \mathrm{~h}$ at $37{ }^{\circ} \mathrm{C}$ and $5 \% \mathrm{CO}_{2}$, the medium was removed and each well was washed with $1 \times$ PBS. The shock treatment was performed at this point. For hypertonic treatment, the cells of each well were exposed to $200 \mu \mathrm{L}$ of glycerol ( 15 or $5 \%, \mathrm{v} / \mathrm{v})$ or $\mathrm{NaCl}(6$ or $2 \%, \mathrm{w} / \mathrm{v})$ in $1 \times$
PBS for $2 \mathrm{~min}$. For hypotonic treatment, cells were exposed to $200 \mu \mathrm{L}$ of DMEM medium diluted with water for $2 \mathrm{~min}$. Each well was washed with $1 \times$ PBS and supplemented with $500 \mu \mathrm{L}$ complete medium for another $42 \mathrm{~h}$ before transfection efficacy analysis. In this study, decompression treatment was defined as the process from hypertonicity to isotonicity (or hypotonicity).

Transfection Efficacy Analysis. The expression of EGFP protein was observed by fluorescent microscopy (Zeiss, Germany) with a $10 \times$ objective. The percentage of EGFPpositive cells and mean fluorescence intensity was quantitatively measured by flow cytometry assay (BD, USA).

Effect of Glycerol Shock Treatment on Endo-Lysosomes. To observe the effect of shock treatment on the intracellular delivery, Hela cells were seeded in $15 \mathrm{~mm}$ glassbottom cell culture dishes (NEST, China) at a density of $2 \times 10^{4}$ cells/well for $24 \mathrm{~h}$. DNA was labeled with YOYO-1 as described previously, $^{23}$ and the $\mathrm{CaP} / \mathrm{DNA}$ precipitate formed, as mentioned above. After cells were incubated with the $\mathrm{CaP} /$ DNA precipitate for $4 \mathrm{~h}$, endo-lysosomes and cell nuclei were stained with LysoTracker Red and Hoechst 33342, respectively. The cells were treated with glycerol or $\mathrm{NaCl}$ shock treatment. The dishes were observed by confocal laser scanning microscopy (CLSM, Zeiss, Germany) with a $40 \times$ objective for changes of the intracellular distribution before and after the shock treatment.

Endo-Lysosomal Leakage Assay. Hela-mAG-Gal3 and 5637-mAG-Gal3 cells were seeded on cell culture dishes for 24 $h$, respectively. Then, endo-lysosomes were stained with LysoTracker Red according to the standard protocol. The changes of endo-lysosomes were observed by fluorescent microscopy or CLSM before and after shock treatment.

Cytotoxicity Analysis. The cytotoxicity of shock treatment or decompression treatment was measured by CCK- 8 assay. Hela cells were seeded in 24-well plates at a density of $5 \times 10^{4}$ cells/well for $24 \mathrm{~h}$. Hela cells were treated with shock treatment in $\mathrm{CaP}$ transfection, as described above. $\mathrm{CaP}$ transfection without shock treatment was used as the control. The CCK-8 assay was performed at $48 \mathrm{~h}$ post-transfection according to wellestablished protocols. The data were given as mean \pm standard deviation based on triplicate repeats.

Chitosan/DNA Delivery. The cells were seeded in 24-well plates as mentioned above. The chitosan solution $(200 \mu \mathrm{g} / \mathrm{mL}$ in $5 \mathrm{mM}$ sodium acetate buffer, $\mathrm{pH} 5.5$ ) was prepared as previously described. ${ }^{24}$ The transfection media for chitosan (DMEM, 5 mM MES, pH 6.5) was prepared for further use. ${ }^{25}$ An equal volume of the plasmid EGFP solution $(100 \mu \mathrm{g} / \mathrm{mL}$ in water) and the chitosan solution were mixed and vortexed for 30 s. Then, chitosan/DNA polyplexes were incubated for $20 \mathrm{~min}$ at $25{ }^{\circ} \mathrm{C}$. The medium in each well was replaced with $500 \mu \mathrm{L}$ of the transfection medium, and chitosan/DNA polyplexes were added into each well at the dose of $1.5 \mu \mathrm{g}$ DNA/well. After incubation for $4 \mathrm{~h}$ at $37^{\circ} \mathrm{C}$ and $5 \% \mathrm{CO}_{2}$, the shock treatment was performed as described above. Then, the cells were washed with $1 \times$ PBS and replaced in complete medium for another $42 \mathrm{~h}$ before transfection efficacy analysis.

\section{ASSOCIATED CONTENT}

\section{Supporting Information}

The Supporting Information is available free of charge at https://pubs.acs.org/doi/10.1021/acsomega.0c04771.

History of glycerol shock treatment in $\mathrm{CaP}$ transfection; cellular uptake of YOYO-1-labeled DNA in Hela cells 
with or without glycerol shock treatment; flow cytometry analysis for $\mathrm{CaP}$ transfection combined with hypertonic shock treatment in Hela cells; and fluorescence images of Hela-mAG-Gal3 cells treated with a $15 \%$ glycerol shock or siramesine treatment (PDF)

\section{AUTHOR INFORMATION}

\section{Corresponding Authors}

Qinghai Shu - School of Material Science and Engineering, Beijing Institute of Technology, Beijing 100081, China; Email: qhshu121@bit.edu.cn

Song Wu - The Affiliated Luohu Hospital of Shenzhen University, Shenzhen University, Shenzhen 518001, China; ○ orcid.org/0000-0003-3504-1630; Email: wusong@ szu.edu.cn

\section{Authors}

Shupeng Wang - School of Material Science and Engineering, Beijing Institute of Technology, Beijing 100081, China; The Affiliated Luohu Hospital of Shenzhen University, Shenzhen University, Shenzhen 518001, China

Shaohua Jin - School of Material Science and Engineering, Beijing Institute of Technology, Beijing 100081, China; (1) orcid.org/0000-0003-4958-4097

Guangzhi Li - The Affiliated Luohu Hospital of Shenzhen University, Shenzhen University, Shenzhen 518001, China

Rui Sun - The Affiliated Luohu Hospital of Shenzhen University, Shenzhen University, Shenzhen 518001, China

Complete contact information is available at:

https://pubs.acs.org/10.1021/acsomega.0c04771

\section{Notes}

The authors declare no competing financial interest.

\section{ACKNOWLEDGMENTS}

This work was supported by the Shenzhen Basic Research Projects from the Shenzhen Science and Technology Innovation Commission (JCYJ20160429172247015 and JCYJ20160429093033251), Shenzhen Key Laboratory Program (ZDSYS20190902092857146), and Special Funds for Strategic Emerging Industries Development in Shenzhen (20180309163446298).

\section{REFERENCES}

(1) Graham, F. L.; van der Eb, A. J. A new technique for the assay of infectivity of human adenovirus 5 DNA. Virology 1973, 52, 456-467.

(2) Jordan, M.; Schallhorn, A.; Wurm, F. M. Transfecting mammalian cells: optimization of critical parameters affecting calcium-phosphate precipitate formation. Nucleic Acids Res. 1996, 24, 596-601.

(3) Jordan, M.; Wurm, F. Transfection of adherent and suspended cells by calcium phosphate. Methods 2004, 33, 136-143.

(4) Batard, P.; Jordan, M.; Wurm, F. Transfer of high copy number plasmid into mammalian cells by calcium phosphate transfection. Gene 2001, 270, 61-68.

(5) Kumar, P.; Nagarajan, A.; Uchil, P. D. Calcium PhosphateMediated Transfection of Eukaryotic Cells with Plasmid DNAs. Cold Spring Harbor Protoc. 2019, 2019, pdb.prot095430.

(6) Ahn, J. Y.; Park, J. Y.; Lim, H. K. Effects of different diluents, cryoprotective agents, and freezing rates on sperm cryopreservation in Epinephelus akaara. Cryobiology 2018, 83, 60-64.

(7) SEARS, E. S. Nonketotic hyperosmolar hyperglycemia during glycerol therapy for cerebral edema. Neurology 1976, 26, 89.

(8) Boya, P. Lysosomal function and dysfunction: mechanism and disease. Antioxid. Redox Signaling 2012, 17, 766-774.
(9) Piao, S.; Amaravadi, R. K. Targeting the lysosome in cancer. Ann. N.Y. Acad. Sci. 2016, 1371, 45.

(10) Seo, S. U.; Woo, S. M.; Lee, H.-S.; Kim, S. H.; Min, K.-j.; Kwon, T. K. mTORC1/2 inhibitor and curcumin induce apoptosis through lysosomal membrane permeabilization-mediated autophagy. Oncogene 2018, 37, 5205 .

(11) Stow, N. D.; Wilkie, N. M. An improved technique for obtaining enhanced infectivity with herpes simplex virus type 1 DNA. J. Gen. Virol. 1976, 33, 447-458.

(12) Frost, E.; Williams, J. Mapping temperature-sensitive and hostrange mutations of adenovirus type 5 by marker rescue. Virology 1978, 91, 39-50.

(13) Gopal, T. V. Gene transfer method for transient gene expression, stable transformation, and cotransformation of suspension cell cultures. Mol. Cell. Biol. 1985, 5, 1188-1190.

(14) Pei, D.; Buyanova, M. Overcoming endosomal entrapment in drug delivery. Bioconjugate Chem. 2018, 30, 273-283.

(15) Stewart, M. P.; Lorenz, A.; Dahlman, J.; Sahay, G. Challenges in carrier-mediated intracellular delivery: moving beyond endosomal barriers. Wiley Interdiscip. Rev.: Nanomed. Nanobiotechnol. 2016, 8, 465-478.

(16) Suwan, K.; Yata, T.; Waramit, S.; Przystal, J. M.; Stoneham, C. A.; Bentayebi, K.; Asavarut, P.; Chongchai, A.; Pothachareon, P.; Lee, K.Y.; Topanurak, S.; Smith, T. L.; Gelovani, J. G.; Sidman, R. L.; Pasqualini, R.; Arap, W.; Hajitou, A. Next-generation of targeted AAVP vectors for systemic transgene delivery against cancer. Proc. Natl. Acad. Sci. U.S.A. 2019, 116, 18571-18577.

(17) D’Astolfo, D. S.; Pagliero, R. J.; Pras, A.; Karthaus, W. R.; Clevers, H.; Prasad, V.; Lebbink, R. J.; Rehmann, H.; Geijsen, N. Efficient intracellular delivery of native proteins. Cell 2015, 161, 674-690.

(18) Pagliero, R. J.; D’Astolfo, D. S.; Lelieveld, D.; Pratiwi, R. D.; Aits, S.; Jaattela, M.; Martin, N. I.; Klumperman, J.; Egan, D. A. Discovery of small molecules that induce lysosomal cell death in cancer cell lines using an image-based screening platform. Assay Drug Dev. Technol. 2016, 14, 489-510.

(19) Aits, S.; Kricker, J.; Liu, B.; Ellegaard, A.-M.; Hämälistö, S.; Tvingsholm, S.; Corcelle-Termeau, E.; Høgh, S.; Farkas, T.; Holm Jonassen, A.; Gromova, I.; Mortensen, M.; Jäättelä, M. Sensitive detection of lysosomal membrane permeabilization by lysosomal galectin puncta assay. Autophagy 2015, 11, 1408-1424.

(20) Gao, D. Y.; Ashworth, E.; Watson, P. F.; Kleinhans, F. W.; Mazur, P.; Critser, J. K. Hyperosmotic Tolerance of Human Spermatozoa: Separate Effects of Glycerol, Sodium Chloride, and Sucrose on Spermolysis. Biol. Reprod. 1993, 49, 112-123.

(21) Armitage, W. J.; Mazur, P. Toxic and osmotic effects of glycerol on human granulocytes. Am. J. Physiol.: Cell Physiol. 1984, 247, C382C389.

(22) Mercier, V.; Larios, J.; Molinard, G.; Goujon, A.; Matile, S.; Gruenberg, J.; Roux, A. Endosomal Membrane Tension Controls ESCRT-III-Dependent Intra-Lumenal Vesicle Formation. bioRxiv 2019, 550483.

(23) Gabrielson, N. P.; Cheng, J. Multiplexed supramolecular selfassembly for non-viral gene delivery. Biomaterials 2010, 31, 91179127.

(24) Mao, H.-Q.; Roy, K.; Troung-Le, V. L.; Janes, K. A.; Lin, K. Y.; Wang, Y.; August, J. T.; Leong, K. W. Chitosan-DNA nanoparticles as gene carriers: synthesis, characterization and transfection efficiency. J. Controlled Release 2001, 70, 399-421.

(25) Lavertu, M.; Méthot, S.; Tran-Khanh, N.; Buschmann, M. D. High efficiency gene transfer using chitosan/DNA nanoparticles with specific combinations of molecular weight and degree of deacetylation. Biomaterials 2006, 27, 4815-4824. 